\title{
Polynomially Adjusted Saddlepoint Density Approximations
}

\author{
Serge B. Provost ${ }^{1} \&$ Susan Z. Sheng ${ }^{1}$ \\ ${ }^{1}$ Department of Statistical and Actuarial Sciences, The University of Western Ontario, London, Canada \\ Correspondence: Serge B. Provost, Department of Statistical and Actuarial Sciences, The University of Western \\ Ontario, London, ON., N6A 5B7, Canada. Tel: 1-519-661-3624. E-mail: provost@ stats.uwo.ca
}

Received: October 8, 2014 Accepted: October 24, 2014 Online Published: December 19, 2014

doi:10.5539/ijsp.v4n1p1 URL: http://dx.doi.org/10.5539/ijsp.v4n1p1

\begin{abstract}
Improved density approximations are obtained by applying certain moment based polynomial adjustments to the saddlepoint approximation. The proposed technique relies on the saddlepoint approximation to the distribution function of a continuous random variable as well. Additionally, a hybrid density estimate is being introduced. Rational functions are also utilized as adjustments. Several numerical examples reveal that, overall, the methodologies that are advocated in this paper consistently produce more accurate distributional approximations.
\end{abstract}

\section{Introduction}

The saddlepoint approximation of a density function was introduced by Daniels(1954). A comprehensive review of this approximating technique as well as related results are available from Reid (1988). Countless applications have been discussed in the statistical and econometric literature over the past few decades. For instance, Kuonen (1999) utilized this technique for approximating the distribution of quadratic forms. The saddlepoint approximation is still finding a variety of new statistical applications, as can been seen for example from the recently published papers of Hyrien et al. (2010), Kolassa and Li (2010), Demaso et al. (2011), Kolassa and Robinson (2011) and Marsh (2011).

The original saddlepoint approximation formulae for the density and distribution functions of a continuous random variable are included in Section 2 where a certain polynomial adjustment to the initial density approximant is derived. A criteria for selecting the degree of the adjustment is also introduced. Seven numerical examples involving single densities and mixture of densities are presented in Section 3; plots and tables of percentiles of the exact as well as the original and adjusted saddlepoint density approximations are also provided for comparison purposes. Adjustments consisting of ratios of polynomial adjustments are discussed in Section 4.

\section{Adjusted Saddlepoint Approximants}

\subsection{The PDF Saddlepoint Approximation}

Daniels (1954) introduced the following saddlepoint approximation of the density function of a continuous random variable $Y$ :

$$
p(y)=\left(\frac{1}{2 \pi K^{\prime \prime}(\hat{\zeta})}\right)^{1 / 2} \exp [K(\hat{\zeta})-\hat{\zeta} y]
$$

where $K(\zeta)$ denotes the cumulant generating function of the random variable of $Y, K^{\prime \prime}()$, its second derivative, and the saddlepoint $\hat{\zeta}$ is the single real solution to the equation $K^{\prime}(\zeta)=y$.

\subsection{The CDF Saddlepoint Approximation}

The Lugannani-Rice approximation (Lugannani and Rice, 1980) to the cumulative distribution function of a continuous random variable $Y$ is given by

$$
\operatorname{Pr}(Y \leqslant y) \approx \Phi(\hat{w})-\phi(\hat{w})\left\{\frac{1}{\hat{v}}-\frac{1}{\hat{w}}\right\},
$$


where $\Phi$ and $\phi$ are respectivley the CDF and PDF of the standard normal distribution,

$$
\hat{w}=\{2[\hat{\zeta} y-K(\hat{\zeta})]\}^{1 / 2} \operatorname{sgn}(\hat{\zeta}),
$$

$\operatorname{sgn}(\cdot)$ denoting the sign function, and

$$
\hat{v}=\hat{\zeta}\left(K^{\prime \prime}(\hat{\zeta})\right)^{1 / 2}
$$

\subsection{Polynomially-Adjusted Saddlepoint Approximation}

Given the moments of a continuous random variables, a polynomially adjusted PDF saddlepoint approximation can be obtained as follows.

Let $Y$ be a continuous random variable defined on the interval $(a, b)$, whose raw moments $E\left(Y^{h}\right)$ are denoted by $\mu_{Y}(h), h=0,1, \ldots$ If the moment generating function is known, the moments can be determined by differentiation and the cumulant generating function $K(t)$ can be obtained by taking logarithm of the moment generating function.

First, the CDF saddlepoint approximation is ultilized to determine an interval that serves as initial support of the approximate distribution. For example, in the case of a continuous random variable having a semi-infinite support, one would initially choose the upper end point $u$ such that the saddlepoint cdf evaluated at $u$ is greater than $1-10^{-15}$. Similarly, when the lower bound of the support of the distribution is unknown, one would initially select the lower end point of the support of the approximate distribution denoted by $l$ to be such that the saddlepoint CDF evaluated at $l$ is less than $10^{-15}$. If the support is infinite, as in the case of the normal distribution, both end points $l$ and $u$ are determined in this manner. Saddlepoint density approximations are then evaluated at multiple points within the interval $(l, u)$ and a second order interpolating spline is fitted to these points. The points of intersection of this spline with the abscissa delimits the support $\left(l^{\star}, u^{\star}\right)$ of the proposed approximation. The interpolant is then normalized so that it integrates to one over $\left(l^{\star}, u^{\star}\right)$ and the resulting approximate density curve is denoted by $\psi(y)$.

On the basis of the first $d$ moments of $Y$, a polynomially adjusted density approximation of the following form is assumed:

$$
g_{d}(y)=\psi(y) \sum_{j=0}^{d} \xi_{j} y^{j},
$$

where $\sum_{j=0}^{d} \xi_{j} x^{j}$ is a polynomial adjustment of degree $d$. The coefficients $\xi_{j}$ are determined by equating the $h^{\text {th }}$ moment of $Y$ to the $h^{\text {th }}$ moment of the approximate distribution specified by $g_{d}(y)$. That is,

$$
\begin{aligned}
\mu_{Y}(h) \quad & =\int_{l^{\star}}^{u^{\star}} y^{h} \psi(y) \sum_{j=0}^{d} \xi_{j} y^{j} \mathrm{~d} y \\
& =\sum_{j=0}^{d} \xi_{j} \int_{l^{\star}}^{u^{\star}} y^{h+j} \psi(y) \mathrm{d} y \\
& \equiv \sum_{j=0}^{d} \xi_{j} m(h+j), \quad h=0,1, \ldots, d,
\end{aligned}
$$

where $m(h)$ denotes the $h^{\text {th }}$ moment associated with $\psi(y)$. This yields a system of linear equations whose solution is

$$
\left(\begin{array}{c}
\xi_{0} \\
\xi_{1} \\
\vdots \\
\xi_{d}
\end{array}\right)=\left(\begin{array}{ccccc}
m(0) & m(1) & \cdots & m(d-1) & m(d) \\
m(1) & m(2) & \cdots & m(d) & m(d+1) \\
\vdots & \vdots & \ddots & \vdots & \vdots \\
m(d) & m(d+1) & \cdots & m(2 d-1) & m(2 d)
\end{array}\right)^{-1}\left(\begin{array}{c}
1 \\
\mu_{Y}(1) \\
\vdots \\
\mu_{Y}(d)
\end{array}\right)
$$

The integrated squared difference between approximations of degrees $\delta$ and $\delta+2$, denoted $\operatorname{ISD}(\delta)$, is proposed as a means of selecting a suitable value for the degree of the polynomial adjustment. Thus,

$$
\operatorname{ISD}(\delta)=\int_{l^{\star}}^{u^{\star}}\left(f_{\delta}(y)-f_{\delta+2}(y)\right)^{2} \mathrm{~d} y
$$


where $l^{\star}$ is the lower bound and $u^{\star}$ is the upper bound of the support of the approximate distribution, is calculated for each value of $\delta$ between 3 and 20. We select the degree $d=\delta+2$ for which the $\operatorname{ISD}(\delta)$ attains a minimum value or reaches a predetermined tolerance level.

\subsection{A Hybrid Approximate Density}

A hybrid density $\tau(y)$ is constructed from the saddlepoint density $\psi(y)$ and its polynomially adjusted form $g_{d}(y)$. Two points, denoted by $r_{a}$ and $r_{b}$, where $\psi(y)$ and $\xi(y)$ intersect, which are for instance located in the neighborhoods of the first and the third quartiles, are determined. Then, the hybrid approximating probability density function is given by

$$
\tau(y)= \begin{cases}\psi(y) & \text { if } y \in\left[0, r_{a}\right) \text { or }\left[r_{b}, 1\right], \\ g_{d}(y) & \text { if } y \in\left[r_{a}, r_{b}\right]\end{cases}
$$

when the support of the distribution is the interval $[0,1]$.

\section{Examples}

Seven density functions are considered in this section, five of which are mixtures. Percentiles of the unadjusted and adjusted approximations are tabulated at various cdf values of the distributions for comparison purposes.

\subsection{A Triangular Density}

Consider the triangular distribution on the interval $(0,2)$, whose density function is $f(x)=x I_{(0,1)}(x)+(2-$ x) $\mathcal{I}_{(1,2)}(x)$, where $\mathcal{I}_{(\cdot)}(x)$ denotes the indicator function. Plots of the exact density and the adjusted and unadjusted saddlepoint approximations are shown in Figure 1. Several percentiles of interest are recorded in Table 1. The bolded values appearing in the tables represent the most accurate percentiles. The integrated squared differences (ISD) between the approximate and exact densities are provided for comparison purposes. The proposed density approximant adjusted by means of a polynomial of degree 16 has the smallest integrated squared difference.

Table 1. Percentiles of the triangular distribution

\begin{tabular}{|c|c|c|c|c|}
\hline & Exact & Saddlepoint (SP) & Adj. SP $(d=8)$ & Adj. SP $(d=16)$ \\
\hline 0.0001 & 0.01414 & 0.01454 & 0.01470 & 0.01404 \\
\hline 0.001 & 0.04472 & 0.04599 & 0.04594 & 0.04437 \\
\hline 0.005 & 0.10000 & 0.10285 & 0.10126 & 0.09917 \\
\hline 0.01 & 0.14142 & 0.14542 & 0.14225 & 0.14032 \\
\hline 0.05 & 0.31623 & 0.32369 & 0.31609 & 0.31583 \\
\hline 0.10 & 0.44721 & 0.45314 & 0.44769 & 0.44794 \\
\hline 0.20 & 0.63246 & 0.63114 & 0.63267 & 0.63316 \\
\hline 0.30 & 0.77460 & 0.76846 & 0.77312 & 0.77384 \\
\hline 0.40 & 0.89443 & 0.88912 & 0.89304 & 0.89344 \\
\hline 0.50 & 1.00000 & 1.00000 & 1.00087 & 1.00042 \\
\hline 0.60 & 1.10557 & 1.11088 & 1.10813 & 1.10676 \\
\hline 0.70 & 1.22540 & 1.23154 & 1.22626 & 1.22510 \\
\hline 0.80 & 1.36754 & 1.36886 & 1.36514 & 1.36663 \\
\hline 0.90 & 1.55279 & 1.54685 & 1.55238 & 1.55378 \\
\hline 0.95 & 1.68377 & 1.67631 & 1.68639 & 1.68310 \\
\hline 0.99 & 1.85858 & 1.85457 & 1.85730 & 1.85960 \\
\hline 0.995 & 1.90000 & 1.89715 & 1.89693 & 1.90086 \\
\hline 0.999 & 1.95528 & 1.95399 & 1.95146 & 1.95425 \\
\hline 0.9999 & 1.98586 & 1.98541 & 1.98407 & 1.98480 \\
\hline ISD & & 0.00090 & 0.00019 & 0.00008 \\
\hline
\end{tabular}




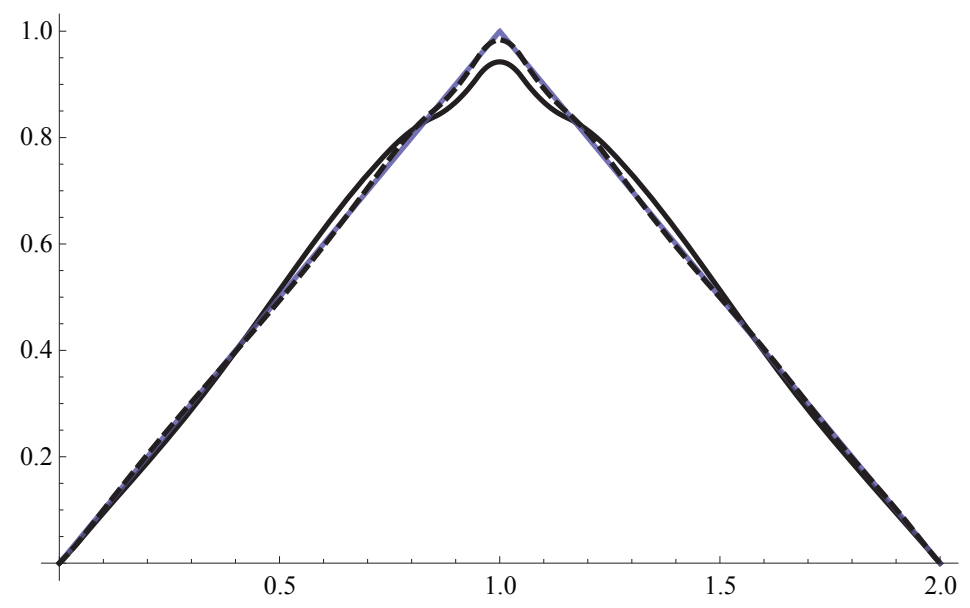

Figure 1. Saddlepoint approximation (dark line) and polynomially adjusted saddlepoint approximation of degree 16 (dashed line) superimposed on the triangular density (grey line)

\subsection{A Weibull Density}

Several exact and approximate percentiles of a Weibull distribution having shape parameter 5 and scale parameter 1 are included in Table 2. The smallest integrated squared difference corresponds to the saddlepoint approximation adjusted with a polynomial of degree 14 .

Table 2. Percentiles of a Weibull distribution

\begin{tabular}{lcccc}
\hline & Exact & Saddlepoint (SP) & Adj. SP $(d=6)$ & Adj. SP $(d=14)$ \\
\hline 0.0001 & 0.15849 & 0.15317 & 0.14311 & $\mathbf{0 . 1 5 6 2 7}$ \\
0.001 & 0.25121 & 0.25456 & $\mathbf{0 . 2 4 9 0 8}$ & 0.25342 \\
0.005 & 0.34675 & 0.34968 & 0.34859 & $\mathbf{0 . 3 4 7 2 8}$ \\
0.01 & 0.39851 & 0.40031 & 0.40055 & $\mathbf{0 . 3 9 8 4 2}$ \\
0.05 & 0.55209 & 0.54970 & 0.55236 & $\mathbf{0 . 5 5 1 9 0}$ \\
0.10 & 0.63758 & 0.63300 & 0.63700 & $\mathbf{0 . 6 3 7 6 7}$ \\
0.20 & 0.74083 & 0.73428 & 0.74012 & $\mathbf{0 . 7 4 0 9 3}$ \\
0.30 & 0.81368 & 0.80645 & 0.81345 & $\mathbf{0 . 8 1 3 7 4}$ \\
0.40 & 0.87429 & 0.86688 & 0.87443 & $\mathbf{0 . 8 7 4 1 9}$ \\
0.50 & 0.92932 & 0.92222 & 0.92984 & $\mathbf{0 . 9 2 9 2 7}$ \\
0.60 & 0.98267 & 0.97610 & 0.98329 & $\mathbf{0 . 9 8 2 6 3}$ \\
0.70 & 1.03782 & 1.03202 & 1.03831 & $\mathbf{1 . 0 3 7 8 3}$ \\
0.80 & 1.09985 & 1.09522 & 1.09989 & $\mathbf{1 . 0 9 9 8 8}$ \\
0.90 & 1.18153 & 1.17886 & 1.18095 & $\mathbf{1 . 1 8 1 7 0}$ \\
0.95 & 1.24538 & 1.24421 & 1.24416 & $\mathbf{1 . 2 4 5 2 7}$ \\
0.99 & 1.35722 & 1.35955 & 1.35690 & $\mathbf{1 . 3 5 6 8 1}$ \\
0.995 & 1.39581 & 1.40005 & 1.39711 & $\mathbf{1 . 3 9 5 5 3}$ \\
0.999 & 1.47186 & 1.47792 & 1.47742 & $\mathbf{1 . 4 7 1 0 3}$ \\
0.9999 & 1.55903 & 1.56944 & 1.57589 & $\mathbf{1 . 5 6 1 3 7}$ \\
\hline ISD & \multicolumn{5}{c}{0.001060} & 0.000064 & 0.000013 \\
\hline
\end{tabular}




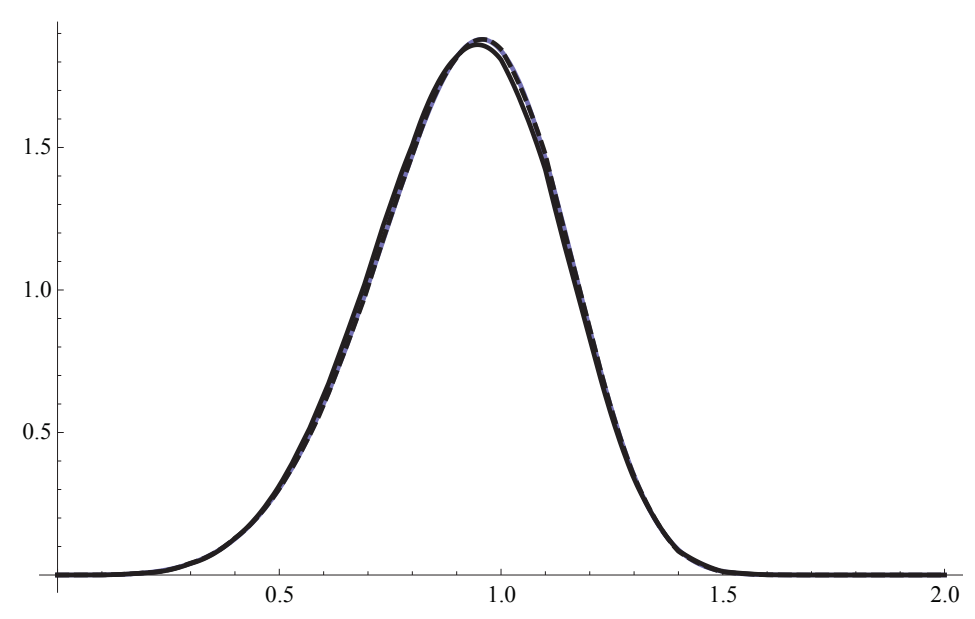

Figure 2. Saddlepoint approximation (dark line) and polynomially adjusted saddlepoint approximation of degree 8 (dashed line) superimposed on the Weibull density (grey line)

\subsection{Mixtures of Beta Densities}

Consider the following mixture of beta density functions: $f_{m}(x)=x(1-x)^{5} /(2 \mathrm{~B}(2,6)) \mathcal{I}_{(0,1)}(x)+x^{6}(1-x)^{2} /(2 \mathrm{~B}(7,3))$ $\mathcal{I}_{(0,1)}(x)$, where $B(a, b)=\frac{\Gamma(a+b)}{\Gamma(a) \Gamma(b)}$, whose density and distribution functions are plotted in Figures 3 and 4 . As can be seen from Table 3, the saddlepoint approximation provides in most cases more accurate percentiles than an initial beta approximation, both being adjusted with a polynomial of degree 4 .

The saddlepoint approximation, its counterpart adjusted with a $4^{\text {th }}$ degree polynomial as well as the corresponding hybrid density approximation are determined for the mixture of beta density functions given by $f_{m}(x)=x(1-$ $x)^{8} /(2 \mathrm{~B}(2,9)) \mathcal{I}_{(0,1)}(x)+x^{6}(1-x)^{2} /(2 \mathrm{~B}(7,3)) \mathcal{I}_{(0,1)}(x)$, and some of the resulting percentiles are tabulated in Table 4. It is seen that, the percentiles obtained from the hybrid density function are the most accurate.

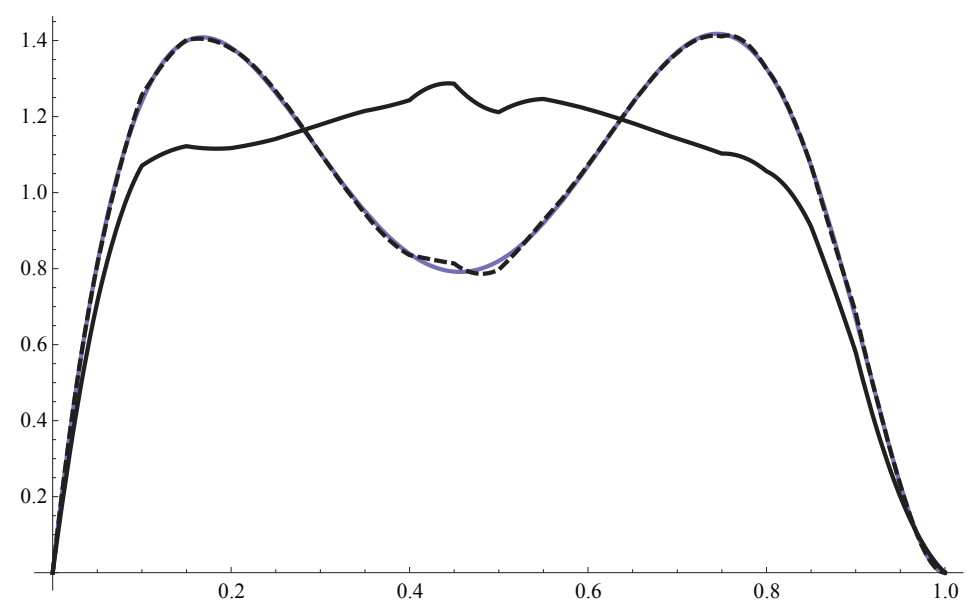

Figure 3. Saddlepoint approximation (line) and polynomially adjusted saddlepoint approximation of degree 16 (dashed line) superimposed on a mixture of beta densities (grey line) 
Table 3. Percentiles of a mixture of beta densities

\begin{tabular}{lccccc}
\hline & Exact & Beta & Adj. B. $(d=4)$ & SP & Adj. SP $(d=4)$ \\
\hline 0.0001 & 0.00310 & 0.00036 & 0.00086 & $\mathbf{0 . 0 0 3 3 7}$ & 0.00356 \\
0.001 & 0.00992 & 0.00246 & 0.00542 & $\mathbf{0 . 0 1 0 7 6}$ & 0.01116 \\
0.005 & 0.02267 & 0.00937 & 0.01748 & $\mathbf{0 . 0 2 4 5 2}$ & 0.02470 \\
0.01 & 0.03259 & 0.01669 & 0.02783 & 0.03522 & $\mathbf{0 . 0 3 4 8 1}$ \\
0.05 & 0.07882 & 0.06406 & 0.07712 & 0.08539 & $\mathbf{0 . 0 7 9 1 4}$ \\
0.10 & 0.11954 & 0.11490 & 0.11985 & 0.13191 & $\mathbf{0 . 1 1 7 2 7}$ \\
0.20 & 0.19150 & 0.20761 & 0.19369 & 0.22135 & $\mathbf{0 . 1 8 9 8 2}$ \\
0.30 & 0.26763 & 0.29542 & 0.27004 & 0.30807 & $\mathbf{0 . 2 7 0 6 5}$ \\
0.40 & 0.36210 & 0.38149 & 0.36248 & 0.39054 & $\mathbf{0 . 3 6 6 5 8}$ \\
0.50 & 0.48313 & 0.46755 & 0.47900 & 0.47893 & $\mathbf{0 . 4 8 1 7 5}$ \\
0.60 & 0.59389 & 0.55501 & 0.59058 & 0.55068 & $\mathbf{0 . 5 8 8 9 1}$ \\
0.70 & 0.67806 & 0.64548 & 0.67827 & 0.63241 & $\mathbf{0 . 6 7 8 2 0}$ \\
0.80 & 0.75024 & 0.74141 & 0.75318 & 0.71846 & $\mathbf{0 . 7 5 4 6 1}$ \\
0.90 & 0.82432 & 0.84811 & 0.82800 & 0.80993 & $\mathbf{0 . 8 2 6 6 6}$ \\
0.95 & 0.87052 & 0.91034 & $\mathbf{0 . 8 7 2 3 7}$ & 0.86201 & 0.86845 \\
0.99 & 0.93116 & 0.97343 & 0.92439 & $\mathbf{0 . 9 2 8 5 5}$ & 0.92479 \\
0.995 & 0.94665 & 0.98424 & 0.93519 & $\mathbf{0 . 9 4 6 4 6}$ & 0.94082 \\
0.999 & 0.96989 & 0.99531 & 0.94694 & 0.97347 & $\mathbf{0 . 9 6 6 8 8}$ \\
0.9999 & 0.98637 & 0.99917 & 0.95046 & 0.99091 & $\mathbf{0 . 9 8 6 7 2}$ \\
\hline ISD & & 0.07633 & 0.00223 & 0.06155 & 0.00005 \\
\hline
\end{tabular}

Table 4. Percentiles of the distribution of another mixture of beta densities

\begin{tabular}{lcccc}
\hline & Exact & Saddlepoint $(\mathrm{SP})$ & Adj. SP $(d=4)$ & Hybrid $(d=4)$ \\
\hline 0.0001 & 0.00212 & 0.00237 & 0.00238 & $\mathbf{0 . 0 0 2 3 7}$ \\
0.001 & 0.00679 & 0.00756 & 0.00751 & $\mathbf{0 . 0 0 7 5 0}$ \\
0.005 & 0.01554 & 0.01722 & 0.01676 & $\mathbf{0 . 0 1 6 7 6}$ \\
0.01 & 0.02238 & 0.02473 & 0.02372 & $\mathbf{0 . 0 2 3 7 2}$ \\
0.05 & 0.05453 & 0.05989 & 0.05438 & $\mathbf{0 . 0 5 4 3 8}$ \\
0.10 & 0.08326 & 0.09295 & 0.08102 & $\mathbf{0 . 0 8 1 0 2}$ \\
0.20 & 0.13513 & 0.16345 & 0.13126 & $\mathbf{0 . 1 3 1 2 6}$ \\
0.30 & 0.19207 & 0.24713 & 0.19514 & $\mathbf{0 . 1 9 5 1 4}$ \\
0.40 & 0.26987 & 0.33340 & 0.28071 & $\mathbf{0 . 2 8 0 7 1}$ \\
0.50 & 0.42224 & 0.42095 & 0.40766 & $\mathbf{0 . 4 0 7 6 6}$ \\
0.60 & 0.58368 & 0.52400 & 0.57535 & $\mathbf{0 . 5 7 5 3 5}$ \\
0.70 & 0.67594 & 0.61059 & 0.67857 & $\mathbf{0 . 6 7 8 5 7}$ \\
0.80 & 0.74977 & 0.71115 & 0.75760 & $\mathbf{0 . 7 5 7 6 0}$ \\
0.90 & 0.82425 & 0.80910 & 0.82704 & $\mathbf{0 . 8 2 7 0 3}$ \\
0.95 & 0.87050 & 0.86159 & $\mathbf{0 . 8 6 6 3 8}$ & 0.86637 \\
0.99 & 0.93116 & $\mathbf{0 . 9 2 8 2 7}$ & 0.91965 & 0.91695 \\
0.995 & 0.94665 & $\mathbf{0 . 9 4 6 2 2}$ & 0.93475 & 0.93383 \\
0.999 & 0.96989 & $\mathbf{0 . 9 7 3 3 2}$ & 0.95947 & 0.94996 \\
0.9999 & 0.98637 & 0.99086 & 0.97893 & $\mathbf{0 . 9 9 0 8 4}$ \\
\hline ISD & & 0.11743 & 0.01265 & 0.01237 \\
\hline
\end{tabular}




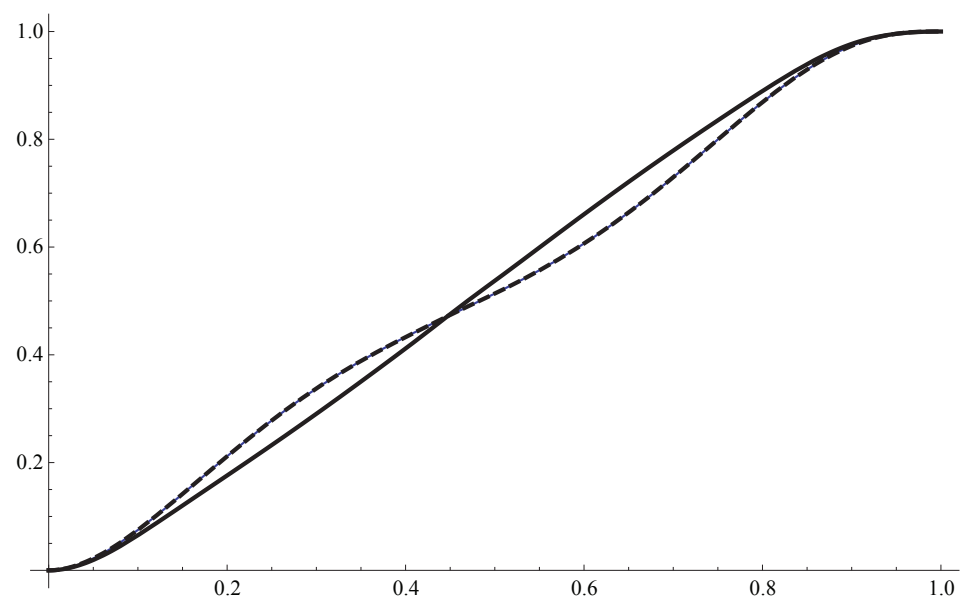

Figure 4. Saddlepoint approximation (dark line) and polynomially adjusted saddlepoint approximation of degree 16 (dashed line) superimposed on the mixture of beta densities (grey line)

\subsection{Mixtures of Normal Densities}

We now consider an equal mixture of two normal densities with parameters $\left(\mu_{1}=-4, \sigma_{1}=4\right)$ and $\left(\mu_{2}=4\right.$, $\sigma_{2}=3$ ), which is plotted in Figure 5 along with the adjusted and unadjusted saddlepoint approximations. As seen from Table 5, the saddlepoint approximation adjusted with a polynomial of degree 12 turns out to be very accurate throughout the support of the distribution.

Table 5. Percentiles of an equal mixture of normal densities

\begin{tabular}{lcccc}
\hline & \multicolumn{1}{c}{ Exact } & Saddlepoint $(\mathrm{SP})$ & Adj. SP $(d=6)$ & Adj. SP $(d=12)$ \\
\hline 0.0001 & -18.1603 & -18.0819 & -19.2254 & $\mathbf{- 1 8 . 1 9 1 6}$ \\
0.001 & -15.5126 & -15.4435 & -15.4579 & $\mathbf{- 1 5 . 4 5 3 1}$ \\
0.005 & -13.3054 & -13.2200 & -13.0624 & $\mathbf{- 1 3 . 3 3 5 2}$ \\
0.01 & -12.2150 & -12.1137 & -12.0682 & $\mathbf{- 1 2 . 2 4 2 1}$ \\
0.05 & -9.12634 & -8.91917 & -9.18863 & $\mathbf{- 9 . 1 1 0 7 2}$ \\
0.10 & -7.36756 & -7.05859 & -7.45214 & $\mathbf{- 7 . 3 6 5 1 4}$ \\
0.20 & -5.02696 & -4.64578 & -5.01122 & $\mathbf{- 5 . 0 3 9 2 0}$ \\
0.30 & -3.08087 & -2.83466 & -2.98508 & $\mathbf{- 3 . 0 7 6 0 8}$ \\
0.40 & -1.19586 & -1.27672 & -1.15275 & $\mathbf{- 1 . 1 8 1 7 9}$ \\
0.50 & 0.57143 & 0.15959 & 0.50925 & $\mathbf{0 . 5 6 5 4 6}$ \\
0.60 & 2.10265 & 1.55117 & 2.02421 & $\mathbf{2 . 0 9 0 4 1}$ \\
0.70 & 3.47695 & 2.96440 & 3.45498 & $\mathbf{3 . 4 7 6 9 4}$ \\
0.80 & 4.86414 & 4.49106 & 4.90744 & $\mathbf{4 . 8 7 4 9 5}$ \\
0.90 & 6.56926 & 6.36664 & 6.62495 & $\mathbf{6 . 5 6 9 6 6}$ \\
0.95 & 7.87045 & 7.74451 & 7.87823 & $\mathbf{7 . 8 5 7 0 5}$ \\
0.99 & 10.1735 & 10.1031 & 10.0668 & $\mathbf{1 0 . 1 8 4 4}$ \\
0.995 & 10.9891 & 10.9275 & 10.8695 & $\mathbf{1 1 . 0 1 4 7}$ \\
0.999 & 12.6420 & 12.5921 & 12.6487 & $\mathbf{1 2 . 6 5 2 1}$ \\
0.9999 & 14.6266 & $\mathbf{1 4 . 5 8 6 9}$ & 15.1917 & 14.5186 \\
\hline ISD & \multicolumn{5}{c}{0.00098} & 0.00009 & $3.91113 \times 10^{-6}$ \\
\hline
\end{tabular}




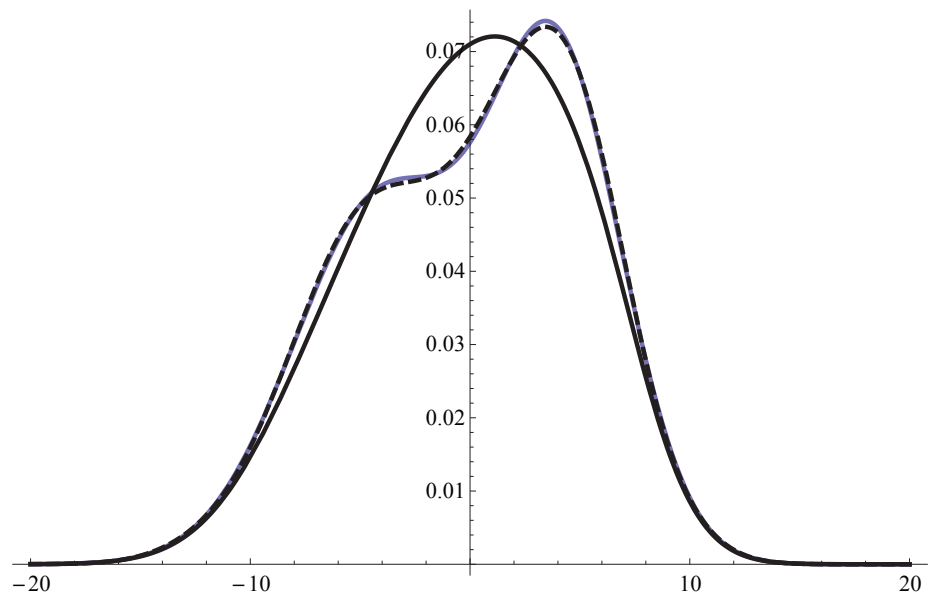

Figure 5. Saddlepoint approximation (dark line) and polynomially adjusted saddlepoint approximation of degree 12 (dashed line) superimposed on the mixture of normal densities (grey line)

Another example, this time taken from Huzurbazar (1999), involves a mixture of two normal densities with parameters $\left(\mu_{1}=4.5, \sigma_{1}=\sqrt{1.2}\right)$ and $\left(\mu_{2}=-2.5\right.$ and $\left.\sigma_{2}=\sqrt{0.8}\right)$ and weights 0.2 and 0.8 . Table 6 contains certain approximate percentiles obtained from unadjusted and adjusted normal and saddlepoint base densities as well as the hybrid approach. We note that the most accurate values are all related to the saddlepoint approximation.

Table 6. Percentiles of Huzurbazar's mixture of normal densities

\begin{tabular}{lcccccc}
\hline & Exact & Nor. $(\mathrm{N})$ & Adj. N. $(d=4)$ & SP & Adj. SP $(d=4)$ & Hybrid \\
\hline 0.0001 & -5.77562 & -9.28504 & -5.77204 & $\mathbf{- 5 . 7 4 0 9 4}$ & -4.46130 & -5.73766 \\
0.001 & -5.20416 & -9.01292 & -5.75231 & $\mathbf{- 5 . 1 6 6 5 4}$ & -4.41613 & -5.16277 \\
0.005 & -4.73402 & -8.25815 & -5.66905 & $\mathbf{- 4 . 6 9 4 8 5}$ & -4.28120 & -4.69047 \\
0.01 & -4.50477 & -7.70420 & -5.57349 & $\mathbf{- 4 . 4 6 2 1 1}$ & -4.17287 & -4.45740 \\
0.05 & -3.87216 & -5.88554 & -4.99656 & -3.81854 & -3.77280 & $-\mathbf{3 . 8 5 1 0 6}$ \\
0.10 & -3.52890 & -4.84403 & -4.48566 & -3.46517 & $\mathbf{- 3 . 5 2 1 1 2}$ & -3.56573 \\
0.20 & -3.10328 & -3.56268 & -3.71237 & -3.01288 & $\mathbf{- 3 . 1 9 2 8 3}$ & -3.21821 \\
0.30 & -2.78500 & -2.63259 & -3.06824 & -2.59344 & $\mathbf{- 2 . 9 3 5 1 6}$ & -2.95454 \\
0.40 & -2.50000 & -1.83581 & -2.46363 & -2.03984 & -2.66872 & $-\mathbf{2 . 6 8 7 3 5}$ \\
0.50 & -2.21500 & -1.09006 & -1.84666 & -1.32671 & $-\mathbf{2 . 3 3 5 5 2}$ & -2.35466 \\
0.60 & -1.89672 & -0.34367 & -1.16052 & -0.45885 & -1.88838 & $\mathbf{- 1 . 9 0 7 7 8}$ \\
0.70 & -1.47110 & 0.45537 & -0.29674 & 0.59507 & -1.22315 & $-\mathbf{1 . 2 4 3 1 9}$ \\
0.80 & 0.82673 & 1.39093 & 1.08361 & 1.93005 & $\mathbf{0 . 6 8 9 6 0}$ & 0.61710 \\
0.90 & 4.50000 & 2.68887 & 3.62995 & 3.78623 & $\mathbf{4 . 6 0 6 6 2}$ & 4.61359 \\
0.95 & 5.23887 & 3.76098 & 5.22115 & 5.01381 & $\mathbf{5 . 4 3 2 9 9}$ & 5.44979 \\
0.99 & 6.30185 & 5.77234 & 7.55897 & 6.23948 & $\mathbf{6 . 2 7 2 6 0}$ & 6.33616 \\
0.995 & 6.64703 & 6.50860 & 8.32931 & 6.59101 & 6.50442 & $\mathbf{6 . 6 1 8 4 5}$ \\
0.999 & 7.32168 & 8.02562 & 9.84422 & $\mathbf{7 . 2 7 6 0 2}$ & 6.86480 & 7.27115 \\
0.9999 & 8.10459 & 9.86658 & 11.5192 & $\mathbf{8 . 0 6 0 4 9}$ & 7.05006 & 8.05876 \\
\hline ISD & & 0.04773 & 0.11059 & 0.06533 & 0.01534 & 0.01511 \\
\hline
\end{tabular}

\subsection{A Mixture of Gamma Densities}

We now considers an equal mixture obtained from a gamma(13,2) density and a gamma(4, 3) density, as plotted in Figure 6. In this case, we made use of both a gamma density and a saddlepoint approximation as base densities. More often than not, the saddlepoint and its adjusted version provide more accurate percentiles than the gamma approximation or its adjusted version. 
Table 7. Percentiles of a mixture of gamma densities

\begin{tabular}{lccccc}
\hline & Exact & Gamma & Adj. G. $(d=4)$ & SP & Adj. SP $(d=4)$ \\
\hline 0.0001 & 0.83461 & 1.03219 & 0.79842 & $\mathbf{0 . 8 4 7 0 1}$ & 0.77090 \\
0.001 & 1.55629 & 1.93950 & 1.51501 & $\mathbf{1 . 5 6 8 0 5}$ & 1.40402 \\
0.005 & 2.46975 & 3.07633 & $\mathbf{2 . 4 3 5 8 9}$ & 2.54813 & 2.29709 \\
0.01 & 3.04871 & 3.78566 & $\mathbf{3 . 0 2 5 5 0}$ & 3.16452 & 2.87388 \\
0.05 & 5.23423 & 6.35401 & $\mathbf{5 . 2 8 2 5 1}$ & 5.39828 & 5.00178 \\
0.10 & 6.88940 & 8.15484 & 7.01175 & 7.17026 & $\mathbf{6 . 7 2 5 6 4}$ \\
0.20 & 9.61455 & 10.7920 & 9.82919 & 10.1256 & $\mathbf{9 . 7 8 2 3 1}$ \\
0.30 & 12.3505 & 13.0296 & $\mathbf{1 2 . 4 9 8 5}$ & 12.7860 & 12.6991 \\
0.40 & 15.3442 & 15.1793 & 15.2124 & $\mathbf{1 5 . 3 1 9 5}$ & 15.5198 \\
0.50 & 18.4065 & 17.3957 & 17.9990 & 17.8366 & $\mathbf{1 8 . 2 9 0 0}$ \\
0.60 & 21.3437 & 19.8199 & 20.8933 & 20.4474 & $\mathbf{2 1 . 0 9 1 7}$ \\
0.70 & 24.2761 & 22.6514 & $\mathbf{2 4 . 0 1 6 5}$ & 23.2962 & 24.0559 \\
0.80 & 27.5426 & 26.2880 & 27.6574 & 26.6770 & $\mathbf{2 7 . 4 7 6 6}$ \\
0.90 & 31.9837 & 31.9276 & 32.6363 & $\mathbf{3 1 . 4 2 3 4}$ & 32.1176 \\
0.95 & 35.6901 & 37.1252 & 36.6161 & $\mathbf{3 5 . 4 0 8 1}$ & 35.9260 \\
0.99 & 42.9329 & 48.2426 & $\mathbf{4 3 . 2 1 2 8}$ & 43.2200 & 43.2344 \\
0.995 & 45.7104 & 52.7711 & 45.0766 & 45.0321 & $\mathbf{4 5 . 6 5 1 0}$ \\
0.999 & 51.6870 & 62.9046 & 47.4860 & $\mathbf{5 1 . 0 3 2 1}$ & 50.4813 \\
0.9999 & 59.4901 & 76.7787 & 48.3247 & $\mathbf{5 6 . 1 3 6 7}$ & 55.6806 \\
\hline ISD & & 0.00185 & $4.1818 \times 10^{-7}$ & 0.00045 & $2.3074 \times 10^{-6}$ \\
\hline
\end{tabular}

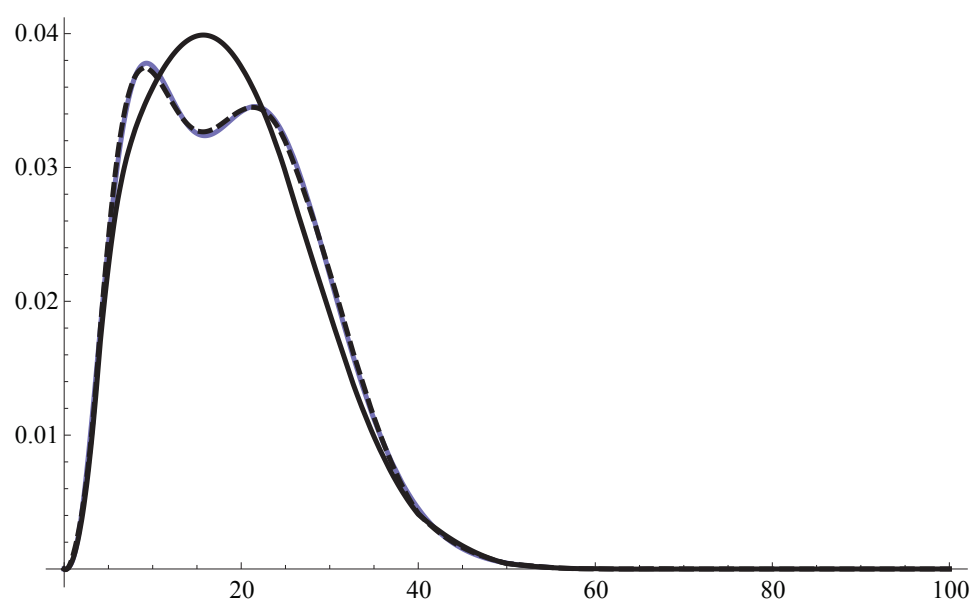

Figure 6. Saddlepoint approximation (dark line) and polynomially adjusted saddlepoint approximation of degree 16 (dashed line) superimposed on the mixture of gamma densities (grey line)

\section{The Use of Rational Functions as Adjustments}

Denoting by $b(y)$ the base density approximating the density function of a continuous random variable $Y$ whose support is $(\alpha, \beta)$, it is assumed that the approximate density has the following form

$$
f_{v, \delta}(y)=b(y) \frac{\sum_{i=0}^{v} a_{i} y^{i}}{\sum_{k=0}^{\delta} c_{k} y^{k}},
$$

where a ratio of polynomials of orders $v$ and $\delta$ is utilized as an adjustment to $b(y)$.

On multiplying both sides of Equation (8) by the denominator of the rational function times $y^{h}$ and integrating over the support of the distribution, one has

$$
\sum_{k=0}^{\delta} c_{k} \int_{\alpha}^{\beta} y^{k+h} f_{v, \delta}(y) \mathrm{d} y=\sum_{i=0}^{v} a_{i} \int_{\alpha}^{\beta} y^{i+h} b(y) \mathrm{d} y,
$$


which is equivalent to

$$
\sum_{k=0}^{\delta} c_{k} \mu_{k+h}=\sum_{i=0}^{v} a_{i} m_{i+h}, \quad h=0,1,2, \ldots, v+\delta,
$$

where the $\mu_{i}$ 's are taken to be the moments of $Y$ and the $m_{j}$ 's, the moments associated with the base density. Letting $c_{\delta}=1$ without any loss of generality, one has

$$
\mu_{\delta+h}+\sum_{k=0}^{\delta-1} c_{k} \mu_{k+h}=\sum_{i=0}^{v} a_{i} m_{i+h} .
$$

On rearranging the terms, one obtains

$$
\sum_{k=0}^{\delta-1} c_{k} \mu_{k+h}+\sum_{i=0}^{v} a_{i}\left(-m_{i+h}\right)=-\mu_{\delta+h}, \quad h=0,1,2, \ldots, v+\delta .
$$

Thus, the required coefficients can be determined from the following equation:

$$
\left(\begin{array}{c}
c_{0} \\
\vdots \\
c_{\delta-1} \\
a_{0} \\
\vdots \\
a_{v}
\end{array}\right)=\left(\begin{array}{cccccc}
\mu_{0} & \cdots & \mu_{\delta-1} & -m_{0} & \cdots & -m_{v} \\
\mu_{1} & \cdots & \mu_{\delta} & -m_{1} & \cdots & -m_{v+1} \\
\vdots & \ddots & \vdots & \vdots & \ddots & \vdots \\
\mu_{\delta+v} & \cdots & \mu_{2 \delta-1+v} & -m_{\delta+v} & \cdots & -m_{\delta+2 v}
\end{array}\right)^{-1}\left(\begin{array}{c}
-\mu_{\delta} \\
-\mu_{\delta+1} \\
\vdots \\
-\mu_{2 \delta+v}
\end{array}\right) .
$$

\subsection{Examples}

First, consider the Weibull distribution as specified in Section 3.2. As shown in Figure 7, the ratio of polynomials adjustment can also produce accurate approximations to its cumulative distribution function.

As can be seen from Figure 8, this is also the case for the mixture of beta $(2,6)$ and beta( $(7,3)$ density functions, which was adjusted by means of a single polynomial in Section 3.3. The cdf resulting from applying a ratio of polynomials as an adjustment to the saddlepoint base density turns out to closely approximate the exact CDF.

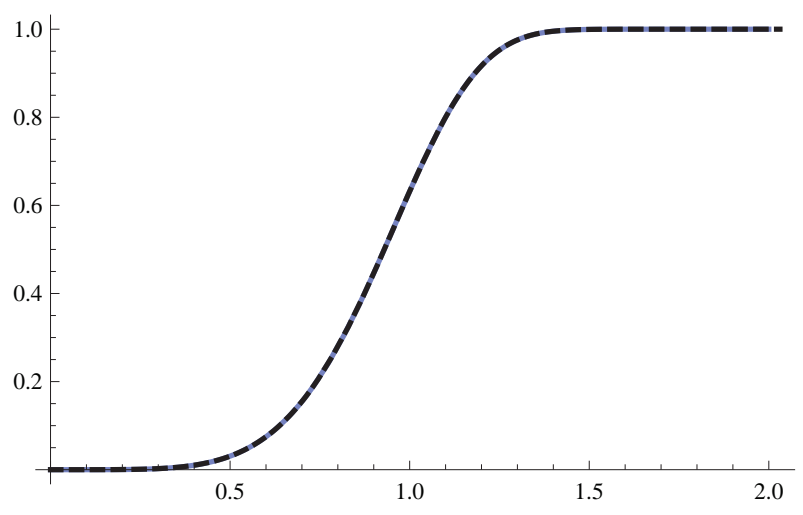

Figure 7. CDF of the saddlepoint approximation adjusted by a ratio of polynomials of degrees 3 and 2 (dashed line) superimposed on the CDF of a Weibull distribution (grey line)

\section{Discussion}

The proposed adjustments to the saddlepoint approximation were shown generally to produce more accurate percentiles throughout the supports of the various distribution that were considered. The improvement is more noticeable in the case of multimodal distributions. The coefficients of the polynomial adjustments are easily determined 
by solving a linear system. All the calculations were carried out with the symbolic computational software Mathematica.

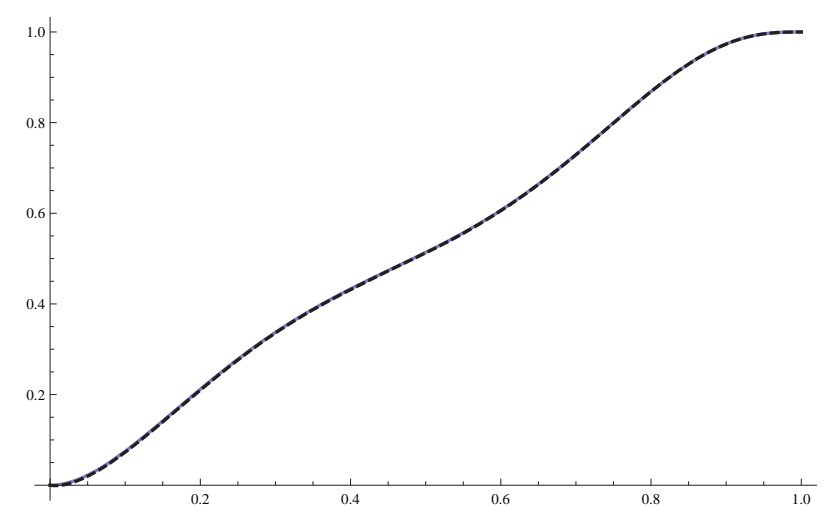

Figure 8. CDF of the saddlepoint approximation adjusted by a ratio of polynomials of degrees 14 and 2 (dashed line) superimposed on the CDF of a mixture of beta densities (grey line)

\section{Acknowledgement}

The financial support of the Natural Sciences and Engineering Research Council of Canada is gratefully acknowledged.

\section{References}

Daniels, H. E. (1954). Saddlepoint approximations in statistics, Annals of Mathematical Statistics, 25, 631-650. http://dx.doi.org/10.1214/aoms/1177728652

Demaso, S. J., Hernandez, F., Brennan, L. A. \& Bingham, R. L. (2011). Application of the simple saddlepoint approximation to estimate probability distributions in wildlife research, The Journal of Wildlife Management, 75(3), 740-746. http://dx.doi.org/10.1002/jwmg.80

Huzurbazar, S. (1999). Practical saddlepoint approximations, The American Statistician, 53, 225-232.

Hyrien, O., Chen, R., Mayer-Proschel, M., \& Noble, M. (2010). Saddlepoint approximations to the moments of multitype age-dependent branching processes, with applications, Biometrics, 66(2), 567-577. http://dx.doi.org/10.1111/j.1541-0420.2009.01281.x

Kolassa, J., \& Li, J. (2010). Multivariate saddlepoint approximations in tail probability and conditional inference, Bernoulli, 16(4), 1191-1207. http://dx.doi.org/10.3150/09-BEJ237

Kolassa, J., \& Robinson, J. (2011). Saddlepoint approximations for likelihood ratio like statistics with applications to permutation tests, The Annals of Statistics, 39(6), 3357-3368. http://dx.doi.org/10.1214/11-AOS945

Kuonen, D. (1999). Saddlepoint approximations for distributions of quadratic forms in normal variables, Biometrika, 86(4), 929-935. http://dx.doi.org/10.1093/biomet/86.4.929

Lugannani, R., \& Rice, S. O. (1980). Saddlepoint approximations for the distribution of sums of independent random variables, Advances in Applied Probability, 12, 475-490. http://dx.doi.org/10.2307/1426607

Marsh, P. (2011). Saddlepoint and estimated saddlepoint approximations for optimal unit root tests, Bootstrap and Numerical Methods in Time Series, 27(5), 1026-1047.

Reid, N. (1988). Saddlepoint methods and statistical inference (with discussion), Statistical Science, 3, 213-238. http://dx.doi.org/10.1214/ss/1177012906

\section{Copyrights}

Copyright for this article is retained by the author(s), with first publication rights granted to the journal.

This is an open-access article distributed under the terms and conditions of the Creative Commons Attribution license (http://creativecommons.org/licenses/by/3.0/). 